\title{
Factorial Validation and Psychometric Properties Establishment of Malay Version Critical Care Family Need Inventory
}

\author{
Dharmalingam $\mathrm{TK}^{\mathrm{a}}$, Kamaluddin $\mathrm{MR}^{\mathrm{b}}$, Hassan $\mathrm{SK}^{\mathrm{a}}$ \\ ${ }^{a}$ Anesthesiology Department, Hospital Universiti Sains Malaysia, Universiti Sains Malaysia, 16150 Kubang \\ Kerian, Kelantan, Malaysia. \\ ${ }^{b}$ School of Psychology and Human Development Studies, Faculty of Social Sciences and Humanities, \\ Universiti Kebangsaan Malaysia, 43600 Bangi, Selangor, Malaysia.
}

\begin{abstract}
Introduction: Despite general acknowledgement of the importance in assessing family needs in critical care patients, there is no psychometric instrument to measure the family needs within Malaysian settings. This study aimed to perform factorial validation and establish psychometric properties of Malay translated Critical Care Family Need Inventory (CCFNI-M) for Malaysians. Methods: This study consisted of four protocols: Forward-Backward translation, validity, internal reliability and inter domain correlations phases. The factorial validation of the CCFNI-M was based on its administration to 109 family members of critical care patients admitted to the Intensive Care Unit of Hospital Universiti Sains Malaysia, Kelantan, Malaysia. At validity phase, factorial validation was performed using Exploratory Factor Analysis using Principal Component Analysis with Varimax rotation. The internal consistency and inter domain correlations were calculated using Cronbach's alpha and Pearson correlation coefficient respectively. Results: Preliminary analyses reported the suitability of data for factorial validation. With reference to the original CCFNI, five factors were extracted which explained $49.4 \%$ of the total variance. After removal of several items for different reasons, the final items in CCFNI-M were 42. The internal consistency values for five dimensions ranged from 0.72 to 0.87 with inter domain correlation values $(r)$ among the dimensions ranged between 0.36 and 0.61 . Conclusion: The high measures of factorial validity, internal consistency and inter domain correlations values of the CCFNI-M make it suitable measure for assessing the family needs of critical care patients.
\end{abstract}

KEYWORDS: Factorial validation, Family member, Critical care patient, Psychometric properties, Malay language

\section{INTRODUCTION}

The first Intensive Care Unit (ICU) in Malaysia was established in $1968 .{ }^{1}$ Since then, intensive care has gained its reputation among medical practitioners and ICUs are now available in all tertiary care hospitals and selected secondary care hospitals under the Ministry of Health. According to $\mathrm{MRCIR}^{1}$, the total numbers of ICU beds in the 49 government hospitals were 600 with a median bed occupancy

Corresponding author:

T. Kumaravadivel Dharmalingam

Anesthesiology Department, Hospital Universiti Sains Malaysia, Universiti

Sains Malaysia, 16150 Kubang Kerian, Kelantan, Malaysia.

Phone: +60162330565

Email: dtkumar123@yahoo.com rate of $90.2 \%$. The same source revealed that there was an increase of $10.5 \%$ (in the year 2013) of cases that seek ICU care compared to the previous year and the total number of cases referred and admitted in ICU for the period of 1968 - 2013 was $37,436 .^{1}$

Intensive Care Units or sometimes referred to as Critical Care Units (CCU) or Intensive Therapy Departments are perceived as the most extreme areas compared to other hospital settings as critically ill patients are being admitted and the most worrying part is the higher mortality rate of these ICU-treated patients. The ICU admissions are usually sudden, with little time for the family members to adjust to the crisis. Patients who seek ICU treatments need constant medical attention which includes utmost level of care, closer 
observation and intensive monitoring by trained and certified medical professionals. The setting and associated environment of ICU seem to be different from the normal or general wards. Generally, ICU wards are considered very expensive, equipped with special beds, high technology instruments with a wide array of equipments and facilities.

An ICU can be a very stressful and daunting environment not only for the patients but also to their respective family members and caregivers. Admission of the family members or close relations into ICU ward can create a great deal of tension and overwhelming anxiety among patient's family members or caregivers. Many authors claimed ICU as a source of anxiety and other psychological distress for the patient's family members. ${ }^{2-4}$

Family members of ICU-treated patients tend to experience high levels of emotional stress such as fear of death, uncertain outcome, financial concerns and disrupted daily routine. Besides triggering devastating psychological distress, the ICU environment also tend to greatly affect the family members ability to interact or provide the best care for the ICU-treated patient. Additionally, ICU admission is often traumatic for the family members which may lead to difficulties in decision making for the patient well-being.

According to Molter ${ }^{5}$, the needs of patient's family members are often neglected by the ICU staff. The needs of the family members are often unnoticed until the family members demonstrate weird and inappropriate coping behaviours at the bed side or until a family member directly seeks assistance in coping. Many studies ${ }^{5-7}$ have highlighted the importance of healthcare providers in providing adequate emotional and physical support to family members by identifying their needs during the crisis. The identification of the needs of family members are considered essential in order to ensure the psychological and physical well-being of the family members as their roles as the primary caregivers are much needed for patients' quality living.

In respect of this, many studies in the West were conducted to investigate the needs of ICU-treated patient's family members or caregivers. The needs of patients' family members were first analysed by Molter ${ }^{5}$ by performing an exploratory descriptive study in which a list of 45 "need" statements developed by Molter from literature reviews and a survey of 23 nursing students were observed. Leske ${ }^{9}$ later developed and established Critical Care Family Needs Inventory (CCFNI) based on Molter's study as reference.

Leske $^{10}$ established the internal psychometric properties and factor analysis of the CCFNI tool with 677 family members over a 9-year period (19801988). The factor validation of CCFNI led to generation of five dimensions: (1) Support, (2) Comfort, (3) Proximity, (4) Information, and (5) Assurance. Since then, CCFNI has gained its popularity and been utilized globally to identify family needs of hospitalized patients. In the context of Malaysia, even though the needs of family members are largely recognized, there is no Malay validated and reliable CCFNI to assess the needs of patient's family members among Malay speaking populations. Care must be taken that those psychometric inventories and instruments that created were in the West may not be adequately addressing the need of local citizens due to language, cultural, geographical and religious differences and barriers.

To date, no local study has been designed to validate and establish psychometric properties of CCFNI in Malaysia. Since the majority of Malaysian populations are Malays and Malay language is the national language, it is extremely crucial to validate CCFNI into Malay language (henceforth, CCFNI-M). Consequently, valid and reliable CCFNI-M is important for two reasons: first, to establish the adequacy of this inventory specially designed to assess the needs of family members in a Malaysia cultural context and second, to further inspire future researches related to the needs of patient's family members.

\section{MATERIALS AND METHODS}

\section{Study design and respondents}

The present validation study adopted cross-sectional design method. The factorial validation study was conducted among family members of ICU-treated patients who were admitted in Hospital Universiti Sains Malaysia, Kelantan, Malaysia due to various illnesses. A series of selection criteria was imposed prior to the recruitment of the respondents. Selection criteria included those who are patient's family members or caregivers, duration of the patient's stay in ICU which was at least for 24 hours, consented and aged 18 years and above. The ethical approval to conduct this factorial validation study was obtained from the ethical committee of Universiti Sains Malaysia, Malaysia. The objective of 
the study was clearly explained to the respondents and issues of confidentiality and anonymity were also clarified and assured. Proper instructions were given and signed consent was obtained prior to respondents' involvement. The average time for questionnaire completion was about 20 minutes. The questionnaire was administered individually and was collected on the same day. The voluntary involvement of the respondents were appreciated and verbally thanked.

\section{Measurement}

The CCFNI is a 45-item inventory which measures the needs of ICU patients' family members and the extent to which they are satisfied. This CCFNI was developed by Molter and Leske ${ }^{11}$, addresses needs related to five dimensions: Information, Assurance, Proximity, Support and Comfort. The Information dimensions consisted of nine items such as "To know which staff members could give what information" and "To know why things were done for a patient". Meanwhile Assurance consisted of seven items such as "To know the expected outcome" and "To know specific facts concerning patient's progress".

In relations to Proximity, there are six items such as "To have visiting hours changed for special conditions" and "To see the patient frequently". Support and Comfort comprised of 14 and 6 items respectively. Examples of items asked in Support are "To have another person with you when visiting critical care unit" and "To talk about the possibility of the patient's death" while items like "To have comfortable furniture in the waiting room" and "To feel accepted by the hospital staff" were designed to measure the Comfort dimension of this instrument.

Each item is scored on a scale of 4-point Likert scale ranging from "not important" to "very important". There was no negative item in this scale which means the higher scores reflect the higher level of that particular need among family members. The internal consistency alpha coefficient of the total CCFNI was $0.92 .{ }^{12}$ The internal consistencies of these five dimensions within CCFNI ranged from 0.88 to $0.98^{12}$ which is considered excellent. In addition, this CCFNI found to be reliable and valid at crosscultural settings. A number of cross-cultural studies $^{13-15}$ acknowledged CCFNI as a valid, reliable and readable tool in assessing the needs of family members of the critical care patients.

\section{Validation protocol}

The present validation study involved several validation protocols in order to establish a valid and reliable Malay version CCFNI.

\section{Forward-Backward Translation}

The study was commenced with forward and backward translation processes. ${ }^{16}$ ForwardBackward translating procedures were carried out to translate CCFNI into Malay language. Three independent bilinguists with psychology, psychiatry and critical care nursing backgrounds were selected for this translating procedure. A professional language proof-reader was hired to endorse the Malay translated CCFNI. Consequently, the Malay version CCFNI was termed as CCFNI-M (the suffix " $M$ " indicates Malay version).

\section{Content and Face validation}

Following the translation processes, the CCFNI-M was subjected to content and face validation. The content validation protocol ensures that the tool includes an adequate and representative set of items that tap the concept investigated. ${ }^{17}$ Often, content validity refers to the appropriateness of the items on the instrument to measure the constructs. ${ }^{18}$ Content validation was often performed by the experts within the field ${ }^{17}$, three experts from psychology, psychiatry and nursing background were assigned to validate the content of the CCFNI-M. The experts reviewed the items in CCFNI-M and ensured that those items were relevant to the scope of measurement within Malaysian context.

While content validity ensures the 'content' aspects of the items, the face validity was carried out among 10 family members to ensure the 'technical' aspects of the questionnaires. Here, the respondents were asked to give feedbacks in terms of layout of the questionnaire, font size, readability and appropriateness of language used in CCFNI-M. After receiving the feedbacks and suggestions from the 10 respondents, a few amendments were made to the CCFNI-M.

\section{Construct and Factorial validation}

Construct validity is defined as the step to assess the degree to which an instrument measures the constructs that it was designed to measure. ${ }^{19}$ For the purpose of construct validation, a total of 109 respondents were recruited in this validation study. The sample size for construct validation phase was based on Gorsuch's ${ }^{20}$ formula in which the total numbers of items in CCFNI were multiplied by 3 to 5. Although the minimum needed sample size was 145 respondents, the present study were only able to recruit 109 respondents. A self-administered 
questionnaire pack which consisted of sociodemography section and CCFNI-M was distributed among the 109 family members of the ICU-treated patients.

\section{Statistical analyses}

Data were analysed using the IBM Statistical Package of Social Sciences (SPSS) version 21.0 Software for descriptive statistics, validity and reliability analyses. Descriptive analyses were done to generate frequency, percentage, mean, standard deviation, and range for socio-demographic information of the respondents. As the step to ensure the sample adequacy of the instrument to proceed with factor analysis, the preliminary analysis for factor analysis was assessed using Kaiser-Meyer-Olkin (KMO) and Bartlett's test of sphericity. ${ }^{21,22}$ Following the preliminary analyses, analyses of factorial validation were carried out.

The factorial validity of items was tested using Exploratory Factor Analysis (EFA) by extracting factors via Principal Component Analysis (PCA) with Varimax rotation. A loading factor of more than plus or minus 0.3 was considered as acceptable. After several runs of factor analysis and removal of items, the best combination was met for factor analysis. The final number of items in each psychometric instrument was subjected to internal reliability testing.

The internal consistency of the CCFNI-M was measured using the Cronbach's Alpha coefficient (a) method. Finally, the inter domain correlations among five dimensions were checked using Pearson correlation coefficient method.

\section{RESULTS}

\section{Socio-demographic profile}

A total of 109 ICU-treated patient's family members participated in this study. The respondents (61 females and 48 males) consisted of 44 parents, 19 relatives, 15 adult children, 14 spouses and siblings, and 3 friends. The family members' average age was 35.39 (SD: \pm 11.54 ) with a range from 19 to 67 years. As for highest educational status, bachelor's degree (47.7\%) and a higher secondary certificate (40.4\%). With regards of duration of stay in ICU, patients were admitted for more than 72 hours (53.2\%) and followed by 24-36 hours stay (26.6\%). In addition, family members claimed this was the first time their family members admitted into ICU (61.5\%). The following Table 1 shows the socio-demographic profiles of the family members.

Table I. Socio-demographic profiles of the patient's family members $(n=109)$

\begin{tabular}{ll}
\hline Socio-demographic profile & $\mathbf{n}(\%)$ \\
\hline Gender & $48(44.0)$ \\
Male & $61(56.0)$ \\
Female & \\
Respondent-patient relationship & $14(12.8)$ \\
Spousal & $44(40.4)$ \\
Children & $15(13.8)$ \\
Parents & $3(2.8)$ \\
Friends & $14(12.8)$ \\
Siblings & $19(17.4)$ \\
Relatives & \\
Educational status & $52(47.7)$ \\
Bachelor degree & $44(40.4)$ \\
Higher secondary & $8(7.3)$ \\
Lower secondary & $5(4.6)$ \\
Primary and below & \\
Duration of stay & $29(26.6)$ \\
$24-36$ hours & $8(7.3)$ \\
$37-48$ hours & $14(12.8)$ \\
$49-72$ hours & $58(53.2)$ \\
More than 72 hours & \\
History of admission & $42(38.5)$ \\
Yes & $67(61.5)$ \\
No &
\end{tabular}




\section{Forward-Backward Translations of CCFNI-M}

The translations processes were considered good and the wordings seemed to be suitable to be used among Malaysians. The backward translated items in CCFNI-M were similar to the original version. A professional language proof-reader resolved word ambiguity issues and ensured the overall suitability of the instrument. Only few amendments were made due to ambiguously worded items and colloquial wordings. The standard agreement among the translators averaged $85.0 \%$ for each item in CCFNI-M.

\section{Content and Face Validity of CCFNI-M}

The experts who reviewed the 'content' aspects of the items in CCFNI-M agreed that the items are relevant and suitable in measuring family needs. The elements of subjectivity in relation to determining content validity were not raised by the experts, indicating the promising content validity of the items in CCFNI-M. With that, it was concluded all the items in CCFNI-M represent all facets of family needs from the context of critical care.

With regards to face validity, respondents acknowledged the readability and understandibility of the CCFNI-M items. Therefore, the language and terms that were used in CCFNI-M were concluded as appropriate and culturally sensitive. However, improvements on font size and layout were made after receiving the feedbacks from the respondents.

\section{Factorial validation of CCFNI-M}

The factorial validation of the CCFNI-M items was assessed using EFA. The factors were extracted using PCA. Prior to factor extraction using PCA, preliminary analyses were conducted to test the suitability of data and also to ensure the sample adequacy for factorial validation.

The preliminary analyses were commenced with the inspection of the Anti-image correlation matrix. The Anti-image correlation matrix for all the items exceeded 0.5 , suggesting favourability of the data set. The KMO value was 0.79 , suggesting adequate sampling to enable factor analysis. Meanwhile, a significant value $(p<0.001)$ of Bartlett's Test of Sphericity also suggested that the data were suitable for factorial validation. The findings of preliminary analyses were found to be acceptable.

Later, Varimax rotation was utilised in order to aid the interpretation of factor loadings. Initial factor analysis was computed by including all the items of CCFNI-M. This resulted in 11 loading factors that explained $68.2 \%$ of the total variance. The scree plot suggested 11 sub components with eigenvalues above 1. Since the theoretical construct of Leske's (1991) suggested five dimensions, the factor analysis was repeated by reducing 11 factors to 5 factors which accounted for $49.4 \%$ of the total variance. The scree plot suggested 5 sub components with eigenvalues above 1 .

After considering the minimum factor loadings of 0.30, item correlation, and the meaningful interpretation of the items; several items were removed. This led to the total number of CCFNI-M which was 42 items. The new KMO value after removal of several items was 0.80 . Bartlett's Test of Sphericity was highly significant $(p<0.001)$. The final factor loadings of 42 items were tabulated in Table 2. All these items showed factor loading of 0.30 and above. Based on the factor loadings matrix, Factor 1 consisted of 11 items, Factor 2 with 12 items, Factor 3 and Factor 4 with 6 items while Factor 5 comprised of 7 items.

Based on the component matrix of final factor loading table above, Factor 1 (Assurance) comprised of 11 items with factor loadings 0.421 to 0.729 . The items are 2, 3, 5, 17, 35, 38, 40, 41, 42, and 43 . Meanwhile, Factor 2 was labelled as Support comprised of 12 items with factor loadings ranging from 0.327 until 0.755 . The items that were placed within Factor 2 were 9, 12, 22, 24, 26, 27, 28, 30, 34 and 37. Only one item (item 9) had factor loading above 0.30 and the remaining 11 items had factor loading above 0.40 .

The next is Factor 3 which is identified as Information. This Information dimension constituted of six items with factor loadings ranged between 0.528 and 0.773 . Item $11,13,15,16,19$, and 25 fall within this dimension. Factor 4 reflects the need for comfort which consisted of six items. Under Comfort, only one item (item 4) had factor loadings above 0.30 and the rest five items (item $6,7,18$, 20 , and 21) exhibited factor loadings exceeding 0.40 .

Finally, Factor 5 which was labelled as Proximity; constituted of seven items with factor loadings ranged between 0.346 until 0.701 . The items that greatly loaded in Proximity are 8, 10, 23, 29, 32, 44 and 45 . Out of these seven items, three items (item 8,29 and 32) had factor loading above 0.30 and the remaining four items $(10,23,44$, and 45$)$ showed factor loadings above 0.40 . 
Table II. Component matrix of Exploratory Factor Analysis of CCFNI-M

\begin{tabular}{|c|c|c|c|c|c|c|}
\hline Original Factor & Items & Component & & & & \\
\hline & & $\begin{array}{l}\text { Factor } 1 \\
\text { (Assurance) }\end{array}$ & $\begin{array}{l}\text { Factor } 2 \\
\text { (Support) }\end{array}$ & $\begin{array}{l}\text { Factor } 3 \\
\text { (Information) }\end{array}$ & $\begin{array}{l}\text { Factor } 4 \\
\text { (Comfort) }\end{array}$ & $\begin{array}{l}\text { Factor 5 } \\
\text { (Proximity) }\end{array}$ \\
\hline$P$ & 41 & 0.729 & & & & \\
\hline A & 43 & 0.686 & & & & \\
\hline$P$ & 40 & 0.681 & & & & \\
\hline A & 35 & 0.643 & & & & \\
\hline A & 42 & 0.633 & & & & \\
\hline A & 5 & 0.626 & & & & \\
\hline I & 8 & 0.568 & & & & \\
\hline$S$ & 2 & 0.456 & & & & \\
\hline A & 17 & 0.456 & & & & \\
\hline A & 1 & 0.427 & & & & \\
\hline I & 3 & 0.421 & & & & \\
\hline $\mathrm{S}$ & 34 & & 0.755 & & & \\
\hline $\mathrm{S}$ & 33 & & 0.694 & & & \\
\hline$S$ & 31 & & 0.693 & & & \\
\hline I & 37 & & 0.648 & & & \\
\hline$S$ & 30 & & 0.622 & & & \\
\hline C & 28 & & 0.560 & & & \\
\hline$S$ & 27 & & 0.550 & & & \\
\hline$S$ & 22 & & 0.514 & & & \\
\hline$S$ & 24 & & 0.471 & & & \\
\hline$S$ & 12 & & 0.437 & & & \\
\hline$S$ & 26 & & 0.401 & & & \\
\hline$S$ & 9 & & 0.327 & & & \\
\hline I & 16 & & & 0.773 & & \\
\hline I & 15 & & & 0.694 & & \\
\hline 1 & 13 & & & 0.657 & & \\
\hline I & 19 & & & 0.644 & & \\
\hline I & 11 & & & 0.561 & & \\
\hline$S$ & 25 & & & 0.528 & & \\
\hline$S$ & 7 & & & & 0.573 & \\
\hline$S$ & 18 & & & & 0.555 & \\
\hline C & 20 & & & & 0.546 & \\
\hline C & 21 & & & & 0.493 & \\
\hline$P$ & 6 & & & & 0.490 & \\
\hline I & 4 & & & & 0.380 & \\
\hline$P$ & 10 & & & & & 0.701 \\
\hline$P$ & 44 & & & & & 0.663 \\
\hline $\mathrm{P}$ & 45 & & & & & 0.615 \\
\hline C & 23 & & & & & 0.417 \\
\hline C & 8 & & & & & 0.387 \\
\hline C & 32 & & & & & 0.368 \\
\hline$P$ & 29 & & & & & 0.346 \\
\hline
\end{tabular}

Note: Extraction Method: Principal Component Analysis. Rotation Method: Varimax with Kaiser Normalization; A: Assurance, S: Support, I: Information, P: Proximity, C: Comfort 
Internal Consistency of CCFNI-M

The internal consistency of the overall CCFNI-M was calculated using the Cronbach's alpha method. The overall internal consistency seemed to be highly promising which is 0.93 . The internal consistency values for 5 dimensions ranged from 0.72 to 0.87 indicating good Cronbach alpha values. Table 3 depicts the internal consistency of the overall scale and also for each dimension in CCFNI-M.

Table III. Internal consistencies of each dimension in CCFNI-M

\begin{tabular}{lll}
\hline Dimension & Number of items & Cronbach alpha (a) \\
\hline Overall & 42 & 0.93 \\
Assurance & 11 & 0.85 \\
Support & 12 & 0.87 \\
Information & 6 & 0.85 \\
Comfort & 6 & 0.72 \\
Proximity & 7 & 0.78 \\
\hline
\end{tabular}

Inter domain correlations of CCFNI-M dimensions

The inter domain correlations revealed positive and significant associations among dimensions of CCFNI-M. The correlation values among the dimensions ranged between 0.36 and 0.61 . The highest correlation value was observed for SupportInformation pair $(r=0.61, p<0.001)$ and followed by
Support-Comfort pair $(r=0.57, p<0.001)$. The least correlation coefficient was evident for AssuranceComfort pair $(r=0.36, p<0.001)$. In summary, all the correlations between CCFNI-M dimensions are within the acceptable to good range. Table 4 depicts the inter domain correlations values among dimensions of CCFNI-M.

Table IV. Inter domain correlations of CCFNI dimensions

\begin{tabular}{llllll}
\hline & Assurance & Support & Information & Comfort & Proximity \\
\hline & & & & & \\
Assurance & 1.00 & $0.37^{*}$ & $0.61^{*}$ & $0.36^{*}$ & $0.44^{*}$ \\
Support & & 1.00 & $0.38^{*}$ & $0.57^{*}$ & $0.62^{*}$ \\
Information & & & 1.00 & $0.46^{*}$ & $0.35^{*}$ \\
Comfort & & & 1.00 & $0.45^{*}$ \\
Proximity & & & & 1.00 \\
\hline
\end{tabular}

Note: ${ }^{*} \mathrm{P}<0.001$

\section{DISCUSSION}

The impact of ICU admission of a family member or close relative can be perceived as traumatic and significantly associated with emotional distress. Previous studies ${ }^{23-28}$ mentioned that family members require some basic needs that must be met in order for them to cope with the ICU admission of their family members and also to adapt themselves with the traumatic and daunting ICU environment. These basic needs of family members were inquired using different research designs and methodologies by targeting the samples of caregivers and family members. In relation to this, obtaining the basic needs via questionnaires was considered useful as it will yield more relevant data as the details or information are directly obtained from the research respondents. ${ }^{29}$
Along this line of thought, many questionnaires and inventories were specifically designed to measure the basic needs of the family members of patients. In ICU settings, the Critical Care Family Need Inventory ${ }^{11}$ has been one of the leading instruments in critical care research for measuring the needs of family members of the critical care patients. It is reminded here, this CCFNI which was created for Western population need to be validated and suited to Malaysian context prior to the administration at local settings. Invalidated questionnaire with foreign language may lead to inaccurate and invalid information due to the cultural, religious and language differences imposed by local population as compared to the Western. This paper sets out to assess the factorial validation and psychometric 
properties of the Malay validated Critical Care Family Need Inventory (CCFNI-M).

In the first part of this validation study, two translation processes were carried out by professional bilinguists. This approach been widely accepted in cross-cultural research. ${ }^{30}$ The outcomes of the translations were accepted with minor amendments made to ambiguously worded and colloquial items which might confuse the respondents. Furthermore, the back translated version of CCFNI-M did not show any contradiction with the original questionnaire, indicating a promising Forward and Backward translations. Overall, the language that was used seemed to be clear, concise and suitable to be administered among Malaysians.

With regards to content validity, the outcomes were considered good based on the agreements and feedbacks from the experts within the acknowledged fields. Content validity refers to the extent to which the items within the questionnaire adequately assess the scope of the measurement. In this study, the content validity phase was carried out as mandatory phase as it is a useful antecedent to examine the construct validity. ${ }^{13}$

As mentioned earlier, the items that listed in CCFNI$M$ seemed to be suitable and relevant to measure the basic needs. No items were removed at this phase as all the items were endorsed as important markers of basic needs. In addition, the content validity of the items in CCFNI was not questionable as the number of studies ${ }^{5,31}$ have been sufficiently established the 'content' of this instrument.

The preliminary analyses for sampling adequacy seemed to be satisfactory and fulfilled all the requirements for sampling adequacy. The KMO values 0.60 were good. ${ }^{22}$ The Bartlett's Test of Sphericity was highly significant suggesting that data is appropriate to proceed with factor analysis. Factor validation was considered very important protocol in clustering the items according to the factors or domains. Hinton et al..$^{32}$ elaborated that construct validation or factorial validation is measured by examining the relationship between the score of different items and uses the correlations between items to specify where the relationship are strong enough to indicate underlying factors.

For the purpose of factorial validation, Principal Component Analysis using Varimax rotation was performed. This is done in order to obtain an interpretable factor clusters. ${ }^{21}$ The EFA with PCA and Varimax rotation showed that the factor loadings of the CCFNI-M items did not correspond to the original CCFNI-M.

While the original version demonstrates five factors, the initial factor analysis in this present study generated 11 factors. This indicated that items were fragmented and did not correlate withthe original CCFNI. In order to reflect the CCFNI subscales, the items were extracted into five scales. The five scales suggested by Leske ${ }^{10}$ are needs for: Support, Comfort, Assurance, Proximity and Information.

The new version of CCFNI-M consisted of 5 factors (Factor 1- Factor 5) as original version, but differs in the distribution and total number of the items in each factor. The factors were labelled based on the number of items that were highly loaded into each factor by referring to the original scale. Hence, Factor 1 was labelled as Assurance, Factor 2 as Support, and Factor 3 as Information. Meanwhile, Factor 4 and Factor 5 were identified as Comfort and Proximity respectively.

During the assessment and inspection of the factor loadings, several items were removed. Several criteria were imposed prior to omission or inclusion of any items within the factors. The criteria include: (a) minimum factor loading of 0.30 , (b) minimal factorial complexity (multiple loading), (c) the internal consistency value of the items corresponding to the overall scale and (d) content of the items.

In addition, it was noted that some items in CCFNI-M were loaded highly on different factors than the original CCFNI. For example, "To talk about feelings about what has happened" was loaded at Support instead of Comfort and "To be called at home about changes in the condition" was loaded at Assurance instead of Proximity. Although such items loaded highly in different factor, the decision to retain or omit were made by consulting few experts within the critical care fields. The cultural contexts and Malaysian perception on the particular needs could be one of the reasons that may explain why certain items in CCFNI-M was highly loaded into different factors compared to the original scale.

The CCFNI-M also proved to have sufficient and promising overall internal consistency value ( $\alpha=$ 0.93). In addition, the internal consistency values of the five dimensions which ranged between 0.72 and 
0.87 showed that the instrument is reliable in measuring the particular dimensions of the family members' needs. According to Peat et al. ${ }^{33}$, a cutoff alpha value of 0.70 is considered sufficient in the field of social science. Most notably, the CCFNI-M showed higher internal consistencies for each scale compared to the previous validation study that conducted among Dutch $(\alpha=0.80)^{13}$ and French speaking population $(\alpha=0.91) .{ }^{34}$

\section{CONCLUSION}

The present validation study was the first study that attempted to validate CCFNI in Malay language. The findings generated in this study presented clear evidence that the CCFNI-M is a valid and reliable measure of needs of family members of critical care patients. It can be concluded that factorial validation and psychometric properties of CCFNI-M were successfully established for the use of Malayspeaking populations. Hence, it is anticipated that administration of CCFNI-M would help to measure needs of Malay speaking family members of critical care patients in a more precise and accurate way.

\section{REFERENCES}

1. Jenny TMG, Tai LL, Tan CC, et al. Malaysian Registry of Intensive Care Report; 2014.

2. Halm MA. Support and reassurance needs: Strategies for practice. Critical Care Nursing Clinics of North America 1992; 4:633-43.

3. McClowry SG. Family functioning during a critical illness: A systems theory perspective. Critical Care Nursing Clinics of North America 1992; 4:559-64.

4. Jamerson PA, Scheibmeir M, Bott M, et al. The experiences of families with a relative in the intensive care unit. Heart Lung 1996; 25:46774.

5. Molter N. Needs of relatives of critically ill patients: a descriptive study. Heart Lung 1979; 8: 332-9.

6. Rose P. The Meaning of Critical Illness to Families. Canadian Journal Nursing Research 1995; 27:83-7.

7. Azoulay E, Pochard F, Chevret S, et al. Meeting the needs of intensive care unit patient families: a multicenter study. Am J Respir Crit Care Med 2001; 163:135-9.

8. Leske JS. Interventions to Decrease Family Anxiety. Crit Care Nurs 2002; 22:61-5.

9. Leske JS. Needs of relatives of critically ill patients: a follow-up. Heart Lung 1986; 15:18993.

10. Molter N, Leske JS. Critical Care Family Needs Inventory, 1983.
11. Molter NC, Leske JS. Critical Care Family Needs Inventory, 1991.

12. Leske JS. Internal psychometric properties of the Critical Care Family Needs Inventory. Heart Lung 1991; 20:236-44.

13. Bijttebier $P$, Delva $D$, Vanoost $S$, et al. Reliability and validity of the Critical Care Family Needs Inventory in a Dutch-speaking Belgian sample. Heart Lung 2000; 29:278-86.

14. Lee L, Lau L. Immediate needs of adult family members of adult intensive care patients in Hong Kong. Journal of Clinical Nursing 2003; 12:490-500.

15. Takman C, Severinsson E. A description of healthcare providers' perceptions of the needs of significant others in intensive care units in Norway and Sweden. Intensive and Critical Care Nursing 2006; 22:228-38.

16. Brislin RW. Back-translation for cross-culture research. Journal of Cross-Cultural Psychology 1970; 1:185-216.

17. McDwermot RJ, Sarvela PD. Health Education Evaluation and Measurement: A practioner"s Perspective. United States: McGraw-Hill componies, Inc., 1999.

18. Nunally JC. Psychometric Theory. New York: McGraw-Hill; 1967.

19. Fayers MP, Machin D. Quality of Life Assesement, Analysis and Interpretation. England: John Wiley \& Sons, Ltd; 2000.

20. Gorsuch RL. Factor Analysis (2nd eds.). Hillsdale, New Jersey: Erlbaum; 1983.

21. Field A. Discovering statistics using SPSS (3rd ed.). SAGE Publication Ltd, London, 2009.

22. Kaiser $\mathrm{H}$. An index of factorial simplicity. Psychometrika 1974; 39: 31-36.

23. Koller PA. Family needs and coping strategies during illness crisis. AACN Clinical Issues in Critical Care Nursing 1991; 2:338-45.

24. Forrester A, Murphy P, Price D, Monaghan J. Critical care family needs: Nurse-family member confederate pairs. Heart and Lung 1990; 19:655-61.

25. Hickey ML, Leske JS. Needs of Families of Critically III Patients: State of the science and Future Directions. Critical Care Nursing Clinics of North America 1992; 4:645-9.

26. Damboise C, Cardin S. Family Centered Critical Care. American Journal of Nursing 2003; 103: 56AA-56EE.

27. Agard A, Harder I. Relatives' experiences in intensive care-Finding a place in a world of uncertainty. Intensive and Critical Care Nursing 2007; 23:170-7.

28. Davidson J. Family Centred Care. Meeting the 
needs of patients' families and helping families adapt to critical illness. Critical Care Nurse 2009; 29:28-34.

29. Brace I. Questionnaire design: How to Plan, Structure, and Write Survey Material for Effective Market Research (2nd eds.). UK: Kogan Page Publishers; 2008.

30. Bernard HR. Research Methods in Cultural Anthropology. Newbury Park, CA: Sage; 1998.

31. Macey BA, Bouman CC. An evaluation of the validity, reliability and readability of the Critical Care Family Needs Inventory. Heart Lung 1991; 20: 398-403.

32. Hinton PR, Brownlow C, MCMurray I, et al. SPSS Explained. Routledge Inc, New York; 2004.

33. Peat J, Mellis C, Williams K, et al. Health Science Research: Handbook of Quantitative Methods. London: Sage; 2002.

34. Coutu-Wakulczyk G, Chartier L. French validation of the Critical Care Family Needs Inventory. Heart Lung 1990; 19: 192-196. 